\title{
Reduced blood-brain leptin signalling in obesity and after slimming: lessons from sheep
}

\author{
Clare L. Adam, Patricia A. Findlay, J. Sally Forno and Anja W. Petrie \\ Rowett Research Institute, Aberdeen Centre for Energy Regulation \& Obesity, Aberdeen, UK
}

The adipose-tissue hormone leptin signals the level of fat in the body and stimulates an anorexigenic response within the hypothalamic region of the brain. However, the brains of obese patients appear resistant to their high circulating concentrations of leptin. It is not clear whether central leptin insensitivity occurs at the level of blood-brain entry or within the hypothalamus itself and it is unknown whether sensitivity is restored on slimming. The present study used a sheep model that mimics the human body in relation to body weight and adiposity, and is amenable to intracerebroventricular (ICV) cannulation. This surgical preparation allows repeated measurement of responses to leptin delivered directly into the hypothalamus to assess intra-hypothalamic sensitivity, and measurement of endogenous leptin concentrations in ventricular cerebrospinal fluid (CSF) and peripheral blood to quantify blood-brain transport in vivo.

Sheep (nine per group; initial body weight 44 (SE 0.6$) \mathrm{kg}$; external adiposity score ${ }^{(1)} 2.25$ ) were surgically prepared with ICV cannulas and either fattened over a 40-week period with ad libitum food (final weight 120 (SE 4.1) kg, adiposity score 4.4 (SE 0.10 ); obese from 20 weeks) or kept on a restricted ration (final weight 65 (SE 1.0) kg, adiposity score 2.1 (SE 0.04); lean). Blood and CSF samples were taken at $14 \mathrm{~d}$ intervals for homologous leptin RIA ${ }^{(2)}$. At 8 -week intervals leptin $(0.5 \mathrm{mg}$ recombinant ovine peptide) was injected ICV and food intake responses were measured. At each time point leptin induced a 30-40\% decrease in voluntary food intake (VFI), providing no evidence for altered intra-hypothalamic leptin sensitivity during developing and sustained obesity. Endogenous plasma leptin concentrations increased fivefold as obesity developed (10.0 (SE 1.53) ng/ml) and continued to increase, twofold, as obesity and high levels of VFI persisted (17.6 (SE 1.28) ng/ml), indicative of central leptin resistance. CSF leptin concentrations increased fivefold as obesity developed (3.5 (SE 0.59) ng/ml) and did not increase thereafter. Thus, the CSF:plasma concentration decreased during persistent obesity, reflecting decreased efficiency of leptin blood-brain transport. Plasma leptin increased marginally over the 40 weeks in lean sheep (3.7 (sE 0.70 ) ng/ $\mathrm{ml}$ ), with a similar magnitude of increase in CSF leptin $(1.4$ (SE 0.20) ng/ml). Thus, lean sheep retained a high CSF:plasma concentration, reflecting efficient leptin blood-brain transport.

Over the next 16 weeks the obese sheep were slimmed on restricted food while the lean sheep were fattened on ad libitum food, with both groups reaching the same end point body weight (102 (SE 1.8) kg) and adiposity score (3.25 (SE 0.05)). At this time point ICV leptin $(0.5 \mathrm{mg})$ reduced VFI in both groups by $40 \%$. Plasma and CSF leptin increased proportionately in fattening sheep. Plasma leptin decreased 2.5-fold in slimming sheep (7.6 (SE 0.71) ng/ml) but a larger, tenfold, decrease in CSF leptin (0.34 (SE 0.08) ng/ml) indicated a further decrease in CSF: plasma concentration and low efficiency of blood-brain transfer.

Thus, apparent central leptin resistance in obesity was not a result of decreased intra-hypothalamic sensitivity but was attributable to decreased efficiency of blood-brain leptin transfer, which developed with increasing leptinaemia. Furthermore, this impairment to leptin blood-brain transport was sustained after $15 \%$ weight loss and decreased leptinaemia. It is concluded that obesity has an adverse effect on leptin entry into the brain that is sustained even after slimming; the hypothalamus continues to receive misleadingly low leptin feedback that would tend to favour orexigenic pathway activation and thereby counteract weight loss.

Work funded by the Scottish Government Rural and Environment Research and Analysis Directorate (RERAD).

1. Marie M, Findlay PA, Thomas L \& Adam CL (2001) J Endocrinol 170, 277-286.

2. Russel AJF, Doney JM \& Gunn RG (1969) J Agric Sci, Camb 72, 451-454. 